\title{
Eleven million adults co-infected with AIDS, TB
}

Eleven million adults are now estimated to be co-infected with HIV and tuberculosis, delegates to the recent 15 th International AIDS Conference learned. Many of these people live in sub-Saharan Africa, where $70 \%$ of those suffering from tuberculosis are also HIV positive, according to Dr. Papa Salif Sow, a professor of infectious diseases at Dakar University in Senegal. HIV suppresses the immune system and increases susceptibility to whatever infections happen to be prevalent. With each active or re-activated case of tuberculosis, HIV worsens.

Sow urged vigorous screening for tuberculosis and active prophylactic treatment for TB of all HIV-positive people, to delay onset of HIV, to reduce morbidity and mortality, and to prevent transmission.

This tactic proved effective in a 2-centre study in Cape Town where isonizide (or a placebo) was given prophylactically to HIV-infected children aged 8 weeks or older. Between January and December 2003, there was a death rate of $14.1 \%$ among those taking the drug, and $76.2 \%$ among the placebo group.

At a prenatal clinic in South Africa, almost $36 \%$ of women are HIV positive, and $60 \%$ of those with active tuberculosis are co-infected with HIV. Their once-per-day observed treatment regimen (DOT) also allows the doctors to treat their HIV.

Dr. Ayesha Khorsany, who operates the clinic at Kwa Zulu Natal, told CMA7 that most new cases of AIDS are in young women. Worldwide, among people younger than 24, girls and women make up nearly twothirds of those living with HIV, the UN reports. In South Africa, Khorsany attributes this to the "sugar-daddy effect." Older men offer girls the 3 Cs: credit cards, a car and cell phone. The myth that having sex with a virgin can cure the disease is also still rampant. The clinic is pursuing an intensive $\mathrm{HIV}$ prevention program, stressing the methods of acquiring HIV/AIDS.

The International AIDS Conference, held July $11-16$ in Bangkok, Thailand, attracted 17000 delegates from 162 countries. The United Nations estimates 38 million people

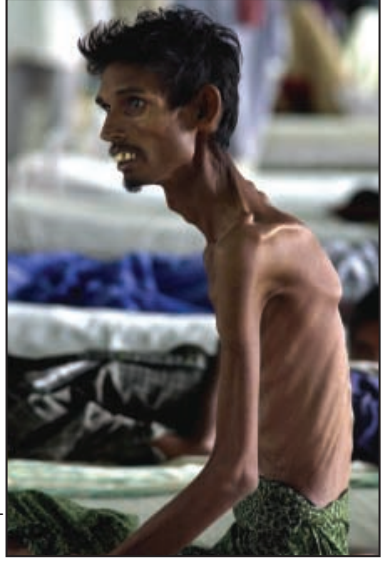

Profile of AIDS: Dkshinamurthy, 40, an HIV-positive patient in India. are now HIV positive; AIDS has killed 20 million people since 1981. - Dr. M. 7. Ferrari, Ottawa

\section{Private Health Care}

\section{Prairie First Nations groups planning 8 private MRI clinics}

At least 8 First Nations are considering establishing for-profit MRI clinics, through agreements with the Canadian Association of Radiologists (CAR).

CAR Chief Executive Normand Laberge is talking with the bands through the Association of First Nations (AFN). He recently negotiated a 5-point agreement with the Muskeg Lake Cree Nation near Saskatoon that will serve as a model.

The agreement launches the radiologists and the $\mathrm{AFN}$ into uncharted territory. Both Health Canada and the Department of Indian Affairs say the provinces of alone have jurisdiction to license First Nations-run diagnostic clinics. Yet the Saskatchewan government has been reluctant to discuss the idea of MRI clinics outside hospitals.

The provincial licence must comply with the Canada Health
Act, which prohibits privately funded delivery of services. Health Canada says it is waiting to see how the provinces respond before taking any action.

The First Nations, meanwhile, insist reserves have jurisdictional autonomy. "There's a definite jurisdictional advantage that hasn't been explored regarding First Nations lands," said Jason Goodstriker, AFN Chief for Alberta.

AFN representatives in Western Canada met July 16-17 near Calgary to discuss the potential for similar clinics in their communities.

First Nations are motivated by the potential for economic development and by a desire to protect universal health care as guaranteed by Aboriginal treaties, says Goodstriker.

"We see this system sliding toward some kind of reduced universal health care," he said. "The only way we can reverse this situation is to become part of the system in terms of offering a solution."

Other bands would consider signing an agreement with CAR, Goodstriker said. "We have to discuss the Canada Health Act and its enforcement on reserves."

The agreement between CAR and Muskeg Lake Cree Nation ensures the facility meets national standards: CAR will inspect the facility, its medical director will report to the association and the clinic will not poach personnel from other Saskatchewan facilities. The deal also stipulates that only medically necessary tests will be performed.

Laberge says CAR is filling a key role in a jurisdictional vacuum. - Amy fo Ebman, Saskatoon 